\title{
Evaluation of a two-step iterative resampling procedure for internal validation of genome-wide association studies
}

\author{
Guolian Kang${ }^{1}$, Wei Liu ${ }^{1}$, Cheng Cheng ${ }^{1}$, Carmen L Wilson², Geoffrey Neale ${ }^{3}$, Jun J Yang ${ }^{4}$, Kirsten K Ness², \\ Leslie L Robison $^{2}$, Melissa M Hudson ${ }^{2}$ and Deo Kumar Srivastava ${ }^{1}$
}

Genome-wide association studies (GWAS) have successfully identified many common genetic variants associated with complex diseases over the past decade. The 'gold standard' method for validating the top single nucleotide polymorphisms (SNPs) identified in GWAS is to independently replicate the findings in similar or diverse large-scale external cohorts. However, for rare diseases, it can be difficult to find an external validation cohort within a reasonable timeframe. In such situations, resampling methods, such as the two-step iterative resampling (TSIR) approach have been used to identify SNPs associated with the outcome of interest. However, the TSIR approach involves choosing several parameters in each step, which can influence the performance of the approach. In this paper, we undertook extensive simulation studies to assess the effect of choice of different parameters on the type I error and power for both binary and continuous phenotypes and also compared the TSIR approach with the traditional one-stage (OS) and two-stage (TS) GWAS analysis. We illustrate the usefulness of the TSIR approach by applying it to a GWAS of childhood cancer survivors. Our results indicate that the TSIR approach with an at least 70:30 split and a cutoff of discovering and replicating SNPs at least 20 times in 100 replications provides conservative type I error control and has near 'optimal' power for internally validated SNPs. Its performance is comparable with the TS GWAS for which an external validation cohort is available with only slight reduction in power in some situations. It has almost the same power as OS GWAS with conservative type I error which leads to fewer false positive findings. TSIR is a powerful and efficient method for identifying and internally validating SNPs for GWAS when independent cohorts for external validation may not be available.

Journal of Human Genetics (2015) 60, 729-738; doi:10.1038/jhg.2015.110; published online 17 September 2015

\section{INTRODUCTION}

Under the common disease common variants hypothesis, genomewide association studies (GWAS) have successfully identified associations between common genetic variants, such as single nucleotide polymorphisms (SNPs) with complex diseases. ${ }^{1-3}$ A two-stage (TS) ${ }^{4}$ or multiple-stage design ${ }^{5,6}$ has been commonly applied to design GWAS to detect SNPs associated with complex diseases. For the TS design, the whole cohort is divided into discovery and replication/validation cohorts. In Stage I, the top signals/SNPs are identified in the discovery cohort using well-defined a priori criterion that are then replicated/ validated in Stage II using an 'independent' replication cohort, that is, independent of the discovery cohort. For multiple-stage designs with more than two stages, after the first stage, Stages II and beyond are usually used to validate the top most significant markers for downstream analyses.

In GWAS involving rare diseases or outcomes in pediatric cancers, we often aim to identify biologic markers that can predict treatment outcomes, help explain treatment-related toxicities or help us understand the effects of treatment modalities on different subtypes of disease. Because these diseases are rare, some with prevalence rates of 1 per million, for example, retinoblastoma and Ewing's Sarcoma, ${ }^{7,8}$ it may not be possible to find an external cohort to validate the top SNPs within a reasonable timeframe. Even when disease outcomes are not rare, it can also be hard to find a suitable external validation cohort. An example is the evaluation of genetic predictors of clinically ascertained outcomes in the SJLIFE cohort, ${ }^{9}$ a study among childhood cancer survivors treated at St. Jude Children's Research Hospital (SJCRH), who have survived 10 or more years from diagnosis and are at least 18 years of age. Because this study includes the largest cohort of childhood cancer survivors with prospective medical/clinical evaluation of health outcomes, it is extremely hard to find another cohort that has similarly ascertained health phenotypes. ${ }^{10}$ In such situations, it is imperative that an innovative and robust internal validation approach is undertaken to validate the top SNPs identified through GWAS. ${ }^{10,11}$

The current research was motivated by a study within the SJLIFE cohort designed to identify the SNPs associated with the obesity

${ }^{1}$ Department of Biostatistics, St. Jude Children's Research Hospital, Memphis, TN, USA; ${ }^{2}$ Department of Epidemiology and Cancer Control, St. Jude Children's Research Hospital, Memphis, TN, USA; ${ }^{3}$ Hartwell Center for Bioinformatics and Biotechnology, St. Jude Children's Research Hospital, Memphis, TN, USA and ${ }^{4}$ Department of Pharmaceutical Sciences, St. Jude Children's Research Hospital, Memphis, TN, USA

Correspondence: Dr DK Srivastava, Department of Biostatistics, St. Jude Children's Research Hospital, Memphis, TN 38105, USA.

E-mail: kumar.srivastava@stjude.org

Received 19 March 2015; revised 14 June 2015; accepted 9 August 2015; published online 17 September 2015 
phenotype (evaluated as a binary outcome measure) in survivors of childhood cancer treated with cranial radiation for which an external cohort to validate our findings was not available. ${ }^{10}$ Thus, we considered an internal validation approach, namely the two-step iterative re-sampling (TSIR) approach, used by Yang et al. ${ }^{11}$ for identifying SNPs associated with the risk of relapse in children treated for acute lymphoblastic leukemia. An alternative approach would be to use a permutation approach, ${ }^{12,13}$ which is particularly suited to situations where the prevalence of the binary outcome is low and the number of cases is small. Another permutation-based internal validation approach is the 'profile significance', ${ }^{14}$ particularly suited for situations where the global level of association between genomic features may be of interest. For large sample sizes, these approaches can be computationally intensive and time consuming. The focus of this manuscript is to describe the operating characteristics of the TSIR approach.

The TSIR approach used by Yang et al. ${ }^{11}$ can roughly be described as follows. The original cohort is split, using a $\pi:(1-\pi)$ ratio, with $\pi=0.5$, into discovery and replication cohorts. Using the discovery cohort, SNPs are individually tested for association with the outcome using Fine and Gray's hazard regression model. All SNPs that are significant at $\alpha_{1}\left(4.4 \times 10^{-3}\right)$ are carried forward to the replication step. A SNP identified in the discovery cohort is considered to be replicated if the same SNP is associated with the outcome in the replication step at $\alpha_{2}(=0.05)$ significance level. This discoveryreplication process is repeated 100 times and a particular SNP is designated as 'associated' or 'internally validated' with the outcome if it is discovered/replicated at least 10 times.

In the approach described by Yang et al., ${ }^{11}$ there was no rationale or statistical justification provided for the following: (i) rationale for the 50:50 split of the original cohort into discovery and replication cohorts, (ii) the choice of $\alpha_{1}=4.4 \times 10^{-3}$ with $\alpha_{2}$ is fixed at level $0.05\left(\alpha_{2}=0.05\right)$, and (iii) a cutoff of 10 in the discovery-replication process. We were interested in assessing how the various choices in (i)-(iii) above affect the statistical properties of the TSIR approach, how the TSIR approach performs for continuous and binary outcomes, and finally how the performance of TSIR approach compares with the one-stage (OS) and TS GWAS analysis?

The research presented here, supported by extensive simulation studies, is designed to guide researchers to use the appropriate choice of parameters when using the TSIR approach for their genomics research involving GWAS when external validation cohorts are not available. The usefulness of the TSIR approach is further demonstrated by applying it to data reported by Wilson et al. ${ }^{10}$

\section{METHODS}

\section{Two-step iterative resampling (TSIR) procedure}

The TSIR described by Yang et al. ${ }^{11}$ was used in the context of survival data. However, in the current analysis, we were interested in binary as well as continuous phenotypes. Accordingly, we discuss the evaluation of binary and continuous end points in parallel.

We assume that, for GWAS in a OS design, there are $N_{0}$ controls and $N_{1}$ cases in a case-control genetic association study (total sample size $N=N_{0}+N_{1}$ ) or $N$ individuals in a genetic association study of a continuous phenotype and that the SNP of interest is biallelic. The two alleles at a SNP are denoted as A and $\mathrm{a}$, where $\mathrm{A}$ is the minor allele and the three genotypes are AA, Aa and aa. Suppose that observations $\left(s_{i}, X_{i}, G_{i}\right), i=1,2, \ldots N$, are available for $N$ individuals, $s_{i}$ is the indicator of case-control status or the quantitative value of the continuous phenotype of the subject $i ; X_{i}=\left[x_{i 1}, x_{i 2}, \ldots, x_{i m}\right]^{\mathrm{T}}$ is the vector of $m$ covariates to be included in the model (for example, demographic or clinical variables); and $G_{i}=0,1$ or 2 is the numerical coding of the three genotypes aa, Aa or AA of the SNP for an individual.

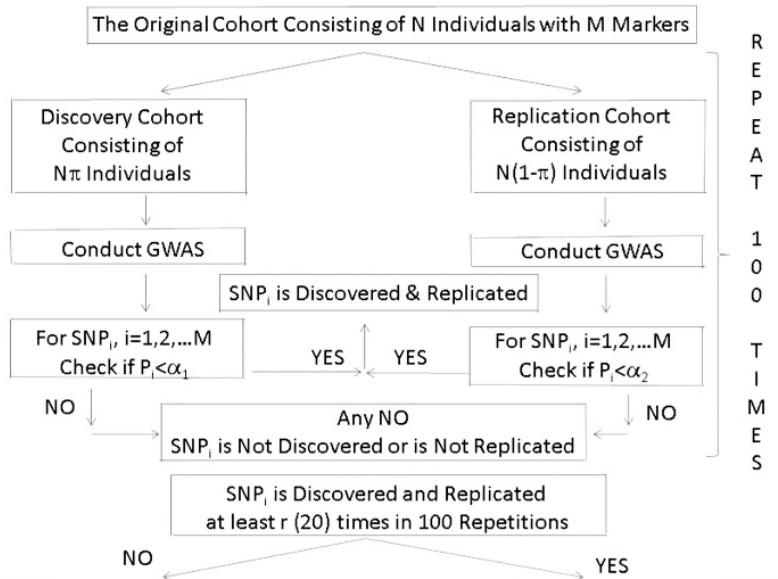

SNP, is not associated with the Phenotype SNP is associated with the Phenotype

Figure 1 The two-step iterative re-sampling procedure for GWAS.

For the TSIR approach, the original cohort is randomly split into discovery and replication set by the ratio $\pi:(1-\pi)$. A SNP is considered discovered and replicated if its association testing $P$-values are statistically significant at levels $\alpha_{1}$ and $\alpha_{2}$ in the discovery and replication steps, respectively (Figure 1). This process is repeated $n=100$ times and a SNP is considered to be 'associated' with the phenotype if the SNP is discovered and replicated at least $r$ times in 100 repetitions.

It may be noted that if we conduct the association analysis, logistic regression or linear regression, with the entire cohort (sample size $N$ ), that is, without splitting the sample into discovery and replication cohorts, then we are conducting traditional OS GWAS. However, without having an independent validation cohort, there is always a concern of false discoveries and the discovered SNPs are subject to suspicion and criticism. In such situations, the proposed TSIR approach overcomes this limitation and the simulation studies suggest that the results based on TSIR approach are more believable and defensible.

\section{Traditional TS GWAS design}

The traditional TS design was introduced as an efficient alternative to conducting a single GWAS analysis (OS design) that includes all genotyped participants. ${ }^{4,15}$ The TS design was proposed as a way to economize on the cost of genotyping, which was quite high when initial GWAS studies were undertaken. In a TS design, $N_{1}^{T S}$ and $N_{2}^{T S}$ are the number of individuals available for genetic analysis in each of the two stages with $N^{T S}=N_{1}^{T S}+N_{2}^{T S}$ being the total sample size. In Stage I, a small set of the individuals $N_{1}^{T S}=N^{T S}{ }^{*} \pi(\pi<0.3, \pi$ is the proportion of participants included in Stage I) would be used as the discovery cohort for whole-genome genotyping and the promising markers at liberal levels of type I error control $\left(\alpha_{1} \geqslant 0.01\right)$ would be identified. Then, in Stage II, a larger cohort of individuals, independent of those in Stage I, of size $N_{2}^{T S}=N^{T S} *(1-\pi)$, with $(1-\pi)>0.7$, would be used as a validation cohort for genotyping the markers selected in Stage I. The final list of markers would be determined based on the results from the Stage II data or in combined Stages I and II data at more stringent levels of type I error control $\left(\alpha_{2}=\alpha / M\right.$, where $M$ is the number of markers associated with phenotype in Stage I and $\alpha$ is the genome-wide significance level). ${ }^{4}$ However, as genotyping costs have decreased over time, the design of TS GWAS has also changed accordingly. Importantly, many more individuals are genotyped for markers spread across the genome in Stage I and tested for association with the phenotype of interest at increasingly stringent level of type I error $\left(\alpha_{1}\right)$, while a more liberal level of type I error control $\left(\alpha_{2}\right)$ is used in Stage II in smaller cohort of individuals, independent of those in Stage I, as the validation cohort. ${ }^{15,16}$ To compare the traditional TS GWAS with the proposed TSIR approach in the current analysis, the parameters for the TS design were chosen to reflect the set-up of the TSIR approach. 


\section{Simulation studies}

We performed extensive simulation studies to evaluate the empirical power and type I error rate of the TSIR procedure for testing associations of SNPs with binary and continuous phenotypes for different parameter combinations as shown below. To evaluate the merits of TSIR, we varied the proportion $(\pi)$ of individuals included in the discovery cohort from 0.3 to 0.9 in increments of 0.1 , and chose $\alpha_{1}=0.01,0.001$ and 0.0001 (with $\alpha_{2}$ fixed at 0.05) for both binary and continuous phenotypes. The prevalence of disease was set at 0.01 , 0.1 and 0.3 for the binary phenotype.

\section{Data generation}

Genotype generations. Given the minor allele frequency (MAF) $p_{\mathrm{A}}$ of minor allele A (major allele a), the genotype frequencies $p(G=g)$ were calculated according to Hardy-Weinberg equilibrium law, that is, $p(G=0)=\left(1-p_{\mathrm{A}}\right)^{2}, p$ $(G=1)=2 p_{\mathrm{A}}\left(1-p_{\mathrm{A}}\right), p(G=2)=\left(p_{\mathrm{A}}\right)^{2}$. Two covariates were considered in our models: $x_{1}$ a binary variable that takes value of 1 with a probability of 0.5 and 0 otherwise, and $x_{2}$ a continuous variable that follows a standard normal distribution. On the basis of these assumed distributions, the complete data on the genotypes and two covariates for a population of 2000000 individuals were generated.

\section{Phenotype generation}

Binary phenotype. The case-control status was determined from the generated genotype and covariate data according to the model similar to that of Kang et al.:17

$$
\operatorname{Pr}\left(s_{i}=1 \mid G_{i}, x_{i 1}, x_{i 2}\right)=\frac{\exp \left(\alpha_{0}+\theta G_{i}+0.5 x_{i 1}+0.5 x_{i 2}\right)}{1+\exp \left(\alpha_{0}+\theta G_{i}+0.5 x_{i 1}+0.5 x_{i 2}\right)} .
$$

We controlled the baseline disease prevalence by setting $\alpha_{0}$ to $0.3,0.1$ and 0.01 to represent high, moderate and low disease prevalence in the case where all three regression coefficients corresponding to SNP, $x_{i 1}$ and $x_{i 2}$ are 0 .

Continuous phenotype. The continuous phenotype was generated from the generated genotype and covariate data according to the model outlined by Wu et al.: ${ }^{8}$

$$
s_{i}=\theta G_{i}+0.5 x_{i 1}+0.5 x_{i 2}+e_{i},
$$

where $e_{i}$ is the random error following a standard normal distribution.

Using the models proposed in (1) and (2), $N_{1}$ cases and $N_{0}$ controls or $N$ samples were randomly generated from the simulated population of 2000000 individuals for binary or continuous outcomes, respectively.

\section{Assessment of type I error probability}

Two values for the MAFs considered were 0.05 and 0.2 in our evaluation of type I error. The case-control status or the continuous phenotype was determined from the generated genotype and covariate data by using their respective models in (1) and (2), with $\theta=0$. To estimate the type I error rate of the TSIR approach, 10000000 replicated datasets were simulated for the case-control model, with 250, 350, 450, 550 and 1000 cases and 1, 2.5 and 4 times the numbers of independent controls under the null hypothesis of $H_{0}: \theta$ $=0$, respectively. The same numbers of replicated datasets were simulated for the continuous phenotype study, with 500, 700, 900, 1100 and 2000 samples under the null hypothesis of $H_{0}: \theta=0$. We used the number of successful replication $r=10,20$ and 25 to estimate the type I error rate of the TSIR procedure. TSIR was applied to each replicate dataset and the empirical type I error rate was estimated as the proportion of replicates in which the tested SNP was identified as 'associated' with the phenotype using TSIR procedure.

\section{Assessment of power}

Three genetic disease models were considered: additive, dominant and recessive with their corresponding genotype codings of $0,1,2 ; 0,1,1$; and $0,0,1$ for three genotypes aa, Aa and AA. The case-control status or the continuous phenotype was determined from the generated genotype and covariate data according to the simulation methods given above, with $\theta=0.2,0.4$ and 0.7 to mimic small, moderate and larger effect sizes, respectively. Datasets were generated 10000 times for each configuration. TSIR used for the type I error simulation was applied to each replicate data-set, and power was estimated as the proportion of replicates in which the tested SNP was identified as 'validated.' On the basis of type I error simulation results, we used $n=20$ in the power estimation of TSIR procedure, as it seemed to control the type I error rate at the desirable levels such as $5 \times 10^{-5}$ or $5 \times 10^{-6}$.

\section{Comparison with the TS design}

To investigate the performance of TSIR, we compared the power of TSIR with that of the TS design under two scenarios based on the different sample sizes. Under the first scenario, the number of individuals in Stage I is the same as those for the TSIR approach. Under the second scenario, it is assumed that we have another independent replication cohort, but the sample sizes in the two stages are similar to the sample sizes in the discovery and replication stages of the TSIR approach. The TS designs under two scenarios are denoted by $\mathrm{TS}_{1}$ and $\mathrm{TS}_{2}$, respectively. To make comparisons reasonable, we selected a significance level combination of $\alpha_{1}=10^{-4}$ and $\alpha_{2}=0.05$ for the TS design to ensure an overall type I error rate per SNP of $5 \times 10^{-6}$ (Table 1). ${ }^{4}$ On the basis of power simulation results above, power was optimized for the TSIR when the ratio of individuals in the discovery and replication cohorts was 70:30 and assuming that both the 'discovery' and 'validation' cohorts were sampled from the same homogenous population. For the TSIR approach, we considered the number of cases for the binary phenotype to be $N_{1}=280,560$ and 1120 and the number of controls to be 2.5 times the number of cases $\left(N_{2}=700,1400\right.$ and 2800), with total sample size of $N=\left(N_{1}+N_{2}\right)=980,1960$ and 3920. For TS1, we considered $N_{1}^{T S}=N$ and $N_{2}^{T S}=\frac{3}{7} N_{1}^{T S}=\frac{3}{7} N\left(N^{T S}=N_{1}^{T S}+N_{2}^{T S}=N+\frac{3 N}{7}=\frac{10 N}{7}\right)$ to be the number of individuals in Stage I (discovery) and Stage II (validation) of the TS design. We then randomly sampled $N_{1}$ and $N_{0}$ individuals $\left(N=N_{1}+N_{0}\right)$ from the general population of 2000000 individuals for the TSIR approach and also used the same sample as the Stage I discovery cohort $\left(N_{1}^{T S}=N=980,1960\right.$ and3920) for the TS analysis. To create a validation cohort for Stage II for the TS approach, we randomly sampled $N_{2}^{T S}=\frac{3}{7} N_{1}^{T S}=420,840$ and 1680 individuals from the general population of 2000000 ; this kept the ratio of participants in the discovery to validation datasets $(0.7: 0.3)$ consistent with the TSIR approach. For $\mathrm{TS}_{2}$, we considered the same sample as that for TSIR but mimicked the features of TS design by splitting $N$ individuals into $N_{1}^{T S}=\frac{7}{10} N$ for Stage I discovery cohort and $N_{2}^{T S}=\frac{3}{10} N$ for Stage II validation cohort, and then applied association analysis methods to these two cohorts.

When considering the continuous phenotype, the number of individuals included in the analyses for the TSIR approach were $N=700,1400$ and 2800. A similar approach was adopted for analysis of the TS design with the continuous phenotype. Datasets were generated 10000 times for each configuration. The power of $\mathrm{TS}_{1}$ and $\mathrm{TS}_{2}$ was estimated as the proportion of replicates in which a SNP was discovered in Stage I at $P<\alpha_{1}$ (where $\alpha_{1}=10^{-4}$ ) and validated in Stage II at $P<\alpha_{2}$ (where $\alpha_{2}=0.05$ ).

The power properties of the TSIR approach were also compared with OS GWAS for simulated $N$ individuals for both binary and continuous outcomes. The power of the OS procedure was estimated as the proportion of replicates in which a SNP was statistically significant at a level of $\alpha_{1} \times \alpha_{2}$.

\section{Simulation results}

Empirical type I error rate of TSIR. Table 1 and Supplementary Table S1 display the empirical type I error rates when $r=20$.

When evaluating the binary phenotype using the TSIR approach, as $\pi$ increased, so did the empirical type I error; however, the type I error was still maintained at a level of $\alpha_{1} \times \alpha_{2}$ per SNP. If $\alpha_{1}=10^{-3}$ and $10^{-4}$, the medians of empirical type one error rate were 0.000033 (range: $0-0.00007$ ) and 0.000001 (range: $0.000000-0.0000056$ ), respectively. The TSIR procedure controlled type I error per SNP at $5 \times 10^{-5}$ and $5 \times 10^{-6}$ if $\alpha_{1}=10^{-3}$ and $10^{-4}$, respectively, which is the same as the type I error control $\left(\alpha_{1} \times \alpha_{2}\right)$ seen in the TS approach. For smaller sample sizes, such as for $N_{1}=250$, it was seen that, irrespective of the prevalence, the type I error rate was much closer to $\alpha_{1} \times \alpha_{2}$ with discovery cohort proportions of $\pi=0.6$ and 0.7 . However, with the discovery cohort proportion of $\pi=0.7$, the type I error rates were much better compared with discovery cohort proportions less than 0.7 , particularly for more stringent values of $\alpha_{1}$, for example, for $\alpha_{1}=0.0001$. The type I error rates corresponding to $\pi=0.60$ and 0.70 are 1.8 and 2.7, 2.8 and 4.2 , and 4.1 and 4.9 corresponding to sample sizes of 250,550 and 1000 , respectively. 
Table 1 The empirical type I error rates $\times 10^{5}$ and $10^{6}$ of TSIR for identifying a CV with MAF of 0.2 associated with the binary and continuous phenotype at $\alpha_{1}=0.001$ and 0.0001 , respectively, and using cutoff of $r=20$ in discovery-replication process

Binary phenotype

$\alpha$

\begin{tabular}{|c|c|c|c|c|c|c|c|c|c|c|c|c|c|}
\hline $\mathrm{N}_{1}$ & $\mathrm{~N}_{0} / \mathrm{N}_{1}$ & $P_{A}$ & Prev. & 0.3 & 0.4 & 0.5 & 0.6 & 0.7 & 0.3 & 0.4 & 0.5 & 0.6 & 0.7 \\
\hline 250 & 1.0 & 0.2 & 0.30 & 0.07 & 1.3 & 3.1 & 3.6 & 3.9 & 0 & 0.00 & 0.6 & 2.1 & 2.3 \\
\hline 250 & 2.5 & 0.2 & 0.30 & 0.18 & 1.9 & 3.5 & 4.0 & 4.3 & 0 & 0.40 & 1.6 & 2.5 & 3.6 \\
\hline 250 & 4.0 & 0.2 & 0.30 & 0.33 & 2.0 & 3.4 & 4.2 & 4.3 & 0 & 0.10 & 1.8 & 3.2 & 4.3 \\
\hline 250 & 1.0 & 0.2 & 0.10 & 0.05 & 1.3 & 3.0 & 3.6 & 3.9 & 0 & 0.10 & 0.6 & 1.7 & 3.0 \\
\hline 250 & 2.5 & 0.2 & 0.10 & 0.20 & 1.6 & 3.2 & 3.9 & 4.2 & 0 & 0.00 & 1.2 & 2.8 & 3.4 \\
\hline 250 & 4.0 & 0.2 & 0.10 & 0.26 & 1.7 & 3.4 & 3.9 & 4.3 & 0 & 0.40 & 1.3 & 2.2 & 2.7 \\
\hline 250 & 1.0 & 0.2 & 0.01 & 0.07 & 1.2 & 2.8 & 3.6 & 3.9 & 0 & 0.00 & 0.8 & 1.8 & 2.7 \\
\hline 250 & 2.5 & 0.2 & 0.01 & 0.26 & 2.0 & 3.7 & 4.4 & 4.7 & 0 & 0.30 & 1.8 & 4.0 & 4.4 \\
\hline 250 & 4.0 & 0.2 & 0.01 & 0.39 & 2.3 & 4.1 & 4.6 & 4.8 & 0 & 0.00 & 1.1 & 3.2 & 3.9 \\
\hline 550 & 1.0 & 0.2 & 0.30 & 0.36 & 1.3 & 2.6 & 3.1 & 3.2 & 0 & 0.00 & 0.2 & 0.6 & 1.3 \\
\hline 550 & 2.5 & 0.2 & 0.30 & 0.25 & 2.1 & 3.8 & 4.4 & 4.6 & 0 & 0.30 & 1.9 & 3.4 & 3.9 \\
\hline 550 & 4.0 & 0.2 & 0.30 & 0.25 & 2.1 & 3.8 & 4.4 & 4.7 & 0 & 0.20 & 2.2 & 2.9 & 4.0 \\
\hline 550 & 1.0 & 0.2 & 0.10 & 0.20 & 1.8 & 3.5 & 4.0 & 4.1 & 0 & 0.10 & 1.3 & 2.3 & 3.3 \\
\hline 550 & 2.5 & 0.2 & 0.10 & 0.32 & 2.2 & 4.0 & 4.5 & 4.9 & 0 & 0.30 & 1.8 & 2.9 & 3.4 \\
\hline 550 & 4.0 & 0.2 & 0.10 & 0.41 & 2.2 & 3.9 & 4.3 & 4.6 & 0.1 & 0.40 & 2.3 & 4 & 5.0 \\
\hline 550 & 1.0 & 0.2 & 0.01 & 0.22 & 1.9 & 3.3 & 4.0 & 4.2 & 0 & 0.30 & 1.7 & 2.8 & 4.2 \\
\hline 550 & 2.5 & 0.2 & 0.01 & 0.42 & 2.5 & 4.2 & 4.7 & 5.0 & 0 & 0.40 & 2.0 & 3.4 & 4.1 \\
\hline 550 & 4.0 & 0.2 & 0.01 & 0.54 & 2.5 & 4.3 & 4.8 & 5.1 & 0 & 0.80 & 2.6 & 3.9 & 4.3 \\
\hline 1000 & 1.0 & 0.2 & 0.30 & 0.24 & 2.0 & 3.9 & 4.5 & 4.6 & 0 & 0.20 & 1.9 & 3.3 & 4.0 \\
\hline 1000 & 2.5 & 0.2 & 0.30 & 0.37 & 2.2 & 3.9 & 4.5 & 4.5 & 0 & 0.40 & 1.9 & 3.6 & 3.6 \\
\hline 1000 & 4.0 & 0.2 & 0.30 & 0.40 & 2.2 & 3.9 & 4.7 & 4.7 & 0 & 0.24 & 1.5 & 3.8 & 4.3 \\
\hline 1000 & 1.0 & 0.2 & 0.10 & 0.32 & 2.0 & 3.7 & 4.3 & 4.5 & 0.1 & 0.20 & 2.0 & 3.3 & 4.6 \\
\hline 1000 & 2.5 & 0.2 & 0.10 & 0.32 & 2.1 & 3.6 & 4.2 & 4.4 & 0 & 0.10 & 1.8 & 3.0 & 4.3 \\
\hline 1000 & 4.0 & 0.2 & 0.10 & 0.35 & 2.4 & 3.9 & 4.5 & 4.8 & 0 & 0.60 & 2.0 & 4.5 & 4.4 \\
\hline 1000 & 1.0 & 0.2 & 0.01 & 0.32 & 2.2 & 3.8 & 4.4 & 4.8 & 0 & 0.50 & 2.2 & 4.1 & 4.9 \\
\hline 1000 & 2.5 & 0.2 & 0.01 & 0.46 & 2.4 & 3.9 & 4.6 & 4.7 & 0 & 0.10 & 2.3 & 3.9 & 4.9 \\
\hline 1000 & 4.0 & 0.2 & 0.01 & 0.42 & 2.6 & 4.3 & 4.7 & 4.9 & 0 & 0.40 & 2.2 & 3.5 & 4.1 \\
\hline
\end{tabular}

Continuous phenotype
0.001

Proportion in discovery cohor

0.0001

Proportion in discovery cohort

\section{3}

3

3.0

4

2.7

.7

.4

3

3.9

4.0

3.3

.4

5.0

4.2

3

4.0

3.6

4.3

4.6

4.3

4.4

4.9

.1

\begin{tabular}{|c|c|c|c|c|c|c|c|c|c|c|c|}
\hline \multicolumn{2}{|l|}{$\alpha_{1}$} & \multicolumn{5}{|c|}{0.001} & \multicolumn{5}{|c|}{0.0001} \\
\hline$N$ & $P_{A}$ & 0.3 & 0.4 & 0.5 & 0.6 & 0.7 & 0.3 & 0.4 & 0.5 & 0.6 & 0.7 \\
\hline 500 & 0.2 & 0.37 & 2.3 & 4.3 & 4.6 & 4.9 & 0.1 & 0.80 & 2.6 & 3.4 & 4.7 \\
\hline 700 & 0.2 & 0.33 & 2.5 & 4.0 & 4.6 & 4.8 & 0 & 0.50 & 2.4 & 3.9 & 4.9 \\
\hline 900 & 0.2 & 0.35 & 2.4 & 4.2 & 4.9 & 5.1 & 0 & 0.20 & 1.5 & 3.6 & 4.3 \\
\hline 1100 & 0.2 & 0.35 & 2.2 & 4.0 & 4.5 & 4.7 & 0 & 0.20 & 2.1 & 3.3 & 4.6 \\
\hline 2000 & 0.2 & 0.39 & 2.2 & 4.0 & 4.4 & 4.7 & 0 & 0.60 & 2.6 & 4.3 & 4.3 \\
\hline 500 & 0.05 & 0.45 & 2.4 & 4.0 & 4.8 & 4.9 & 0.12 & 0.47 & 3.1 & 5.2 & 6.0 \\
\hline 700 & 0.05 & 0.45 & 2.3 & 3.9 & 4.6 & 4.9 & 0 & 0.80 & 2.5 & 3.7 & 4.0 \\
\hline 900 & 0.05 & 0.52 & 2.7 & 4.6 & 5.3 & 5.4 & 0 & 0.70 & 2.7 & 5.1 & 5.6 \\
\hline 1100 & 0.05 & 0.37 & 2.3 & 3.9 & 4.5 & 4.7 & 0 & 0.50 & 1.7 & 3.0 & 3.6 \\
\hline 2000 & 0.05 & 0.46 & 2.4 & 4.4 & 5.1 & 5.2 & 0 & 0.50 & 1.9 & 3.4 & 3.9 \\
\hline
\end{tabular}

Abbreviations: CV, common variants; MAF, minor allele frequency; TSIR, two-step iterative resampling.

It is seen that as the sample size increases, the type I error control improves and the impact of the proportion of individuals allocated to the discovery stage relative to the validation stage is minimal when the type I error control used in the discovery phase is somewhat larger $\alpha_{1} \geqslant 0.001$. But, for more stringent values of $\alpha_{1}$, such as $\alpha_{1}=0.0001$, the TSIR approach with $70 \%$ in the discovery cohort still provides qualitatively superior type I error control. From Table 1, similar conclusions can be drawn when the phenotype is continuous.

The type I error rate per SNP was not maintained at $\alpha_{1} \times \alpha_{2}$ level when $r=10$ was chosen as the validation cutoff (Supplementary Table S2). Similarly, the type I error rate per SNP was too conservative when $r=25$ was chosen as 
a $M A F=0.2 ; \operatorname{prev}=0.3 ; \theta=0.2$

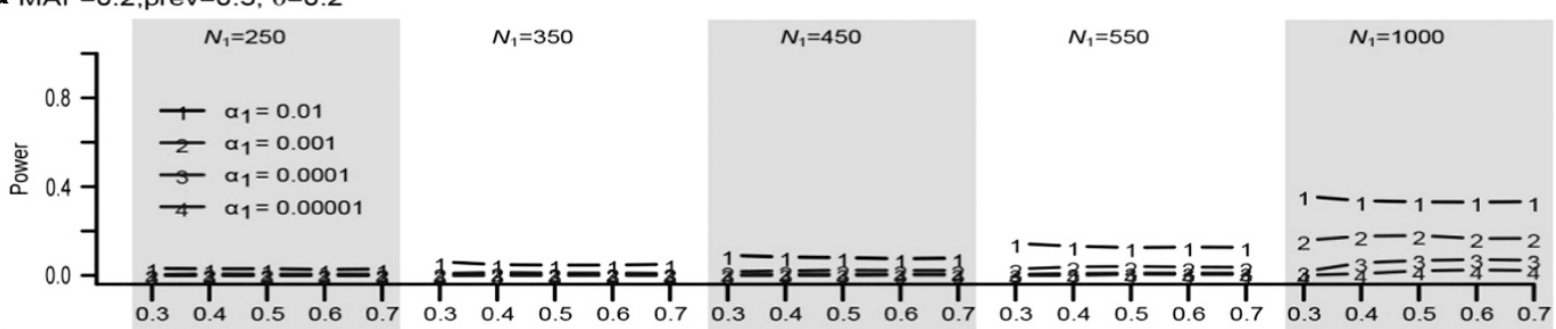

b $M A F=0.2 ;$ prev $=0.3 ; \theta=0.4$

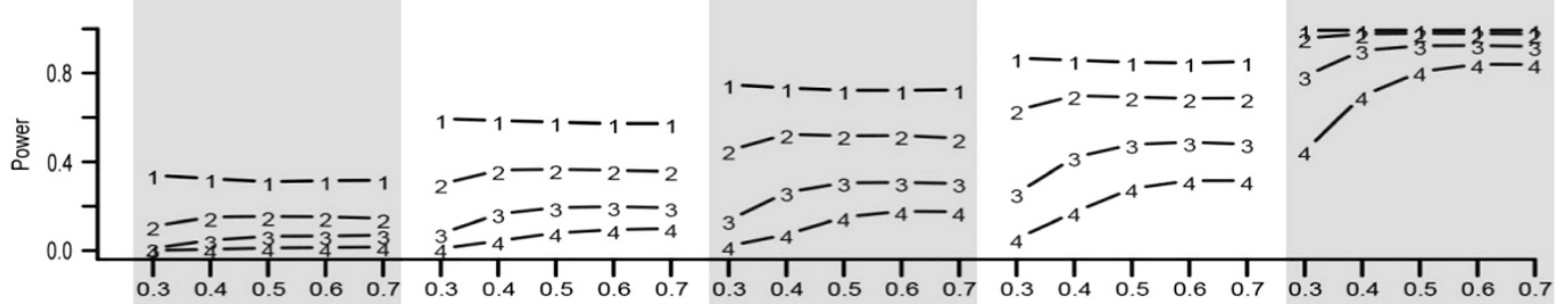

C $M A F=0.2 ;$ prev $=0.3 ; \theta=0.7$

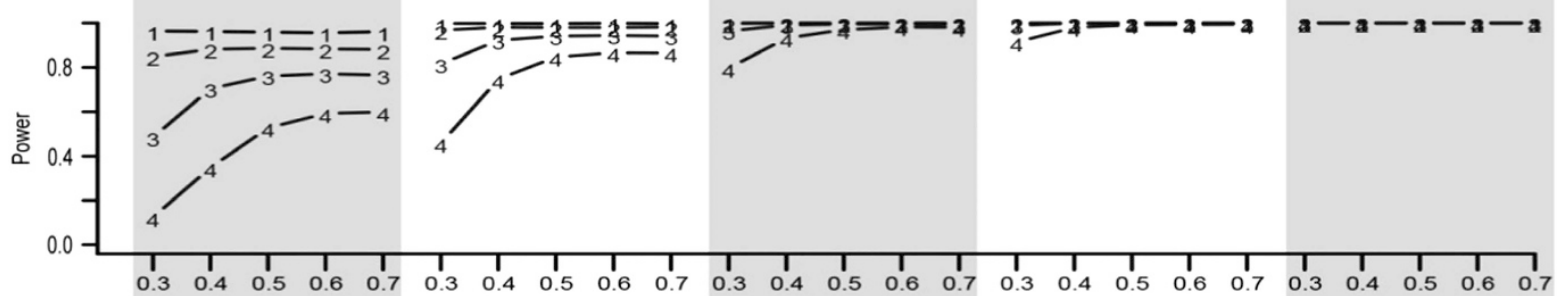

d $\mathrm{MAF}=0.2 ;$ prev $=0.01 ; \theta=0.2$

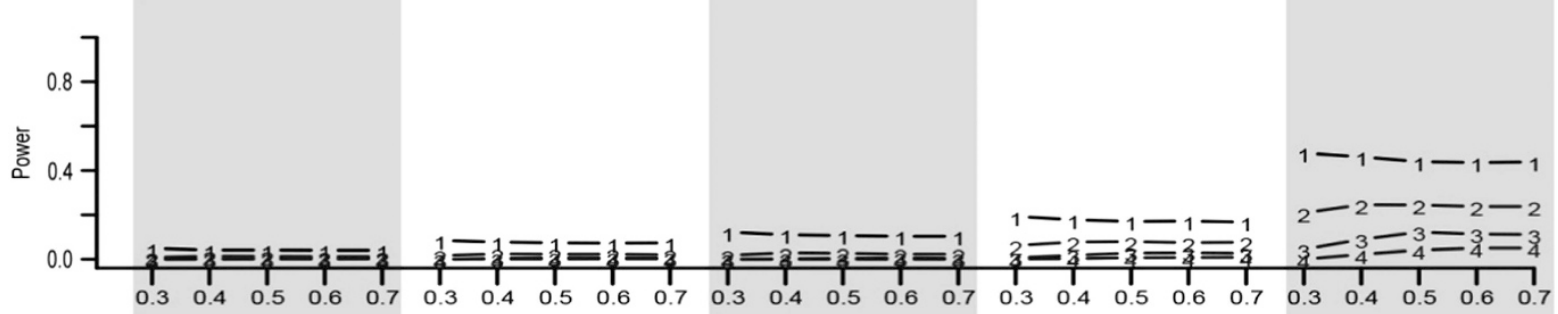

e MAF $=0.2$; prev $=0.01 ; \theta=0.4$

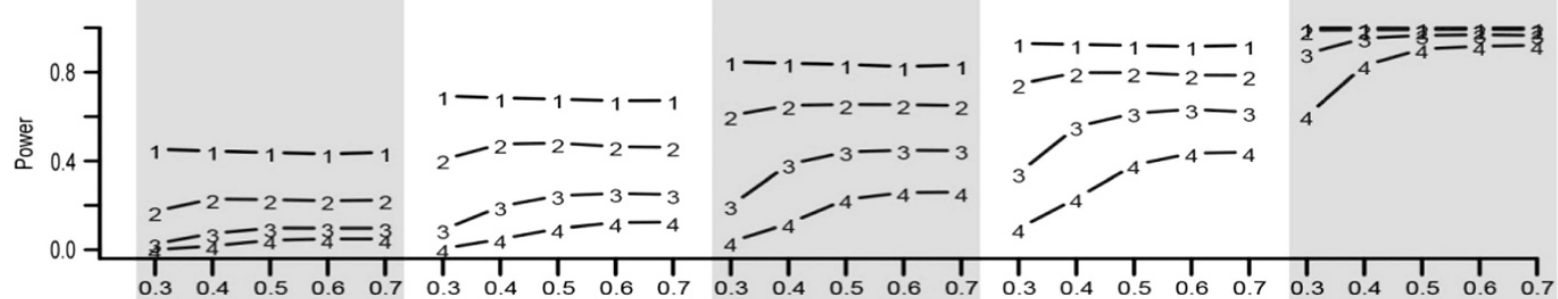

f $\mathrm{MAF}=0.2$; prev $=0.01 ; \theta=0.7$

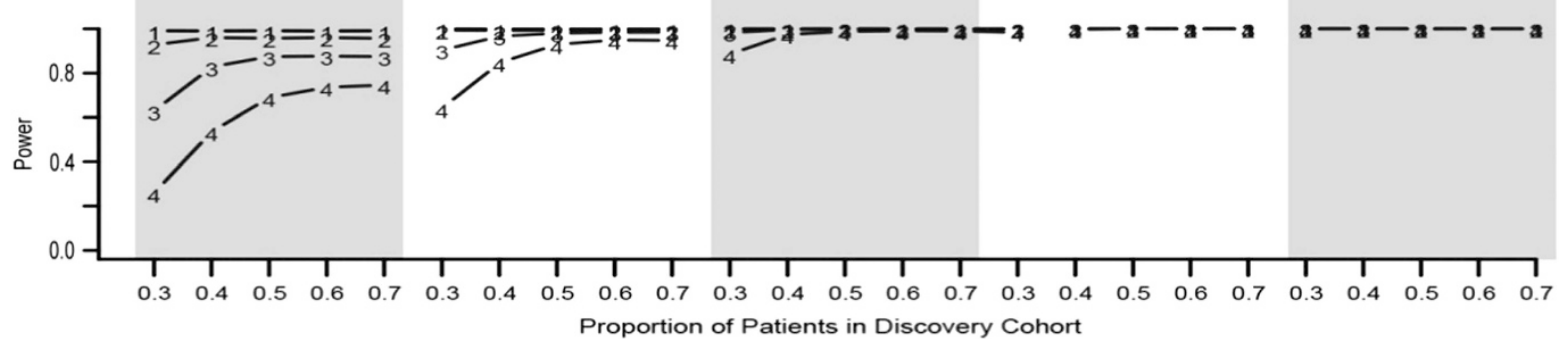

Figure 2 Empirical power of TSIR for detecting a SNP with a MAF of 0.2 for a binary phenotype. a-c are for small effect size $\theta=0.2$, moderate effect size 0.4 , and large effect size 0.7 for a large prevalence of 0.3 , respectively. $\mathbf{d}-\mathbf{f}$ are for small effect size $\theta=0.2$, moderate effect size 0.4 , and large effect size 0.7 for a small prevalence of 0.01 , respectively. The solid lines with the numbers of $1-4$ correspond to $\alpha_{1}=0.01,0.001,0.0001$ and 0.00001 , respectively. 
the validation cutoff (Supplementary Table S3). In addition, with the discovery cohort proportion of $\pi=0.8$ and 0.9 , the type I error rates were close to those when $\pi=0.7$ for $\alpha_{1}>0.0001$, but were a little higher than those when $\pi=0.7$ for $\alpha_{1}=0.0001$ (Supplementary Table S3). However, the power when $\pi=0.7$ for all simulated $\alpha_{1}$ plateaued (see below for empirical power). Thus, for power estimation or comparison, we will plot the results for $\pi$ up to 0.7 .

Empirical power of TSIR. All power evaluations, discussed below, were conducted using $r=20$ as the validation cutoff. On the basis of the extensive power simulation studies, the following conclusions can be drawn:

Binary phenotype. From Figures 2 and 3 and Supplementary Figures S1 and S2, it is seen that when $\alpha_{1}=0.01$, the power of the TSIR approach for detecting a SNP with a MAF of 0.2 is not affected by the proportion of individuals $(\pi)$ included in Stage I (discovery cohort). However, as expected, for more conservative values of $\alpha_{1}$, that is, $\alpha_{1} \leqslant 10^{-3}$, the power of TSIR approach first increased sharply and then plateaued with the increasing values of $\pi$. Also, not surprising, as $\alpha_{1}$ became more conservative, the power of TSIR approach decreased.

When both the effect size of the SNP and the sample size were small or very large, the proportion of individuals included in discovery cohort had little effect on the power of the TSIR approach. In contrast, if the effect size was moderate or small but the sample size was large, or the effect size was large but the sample size was small, then the power estimates were optimized when $\pi$ ranged between 0.5 and 0.7. However, for stringent values of $\alpha_{1}, \pi=0.7$ for the discovery cohort provided consistently better power. Neither the prevalence of disease nor the MAF affected the power of the TSIR approach (Supplementary Figures S1 and S2)

Continuous phenotype. As seen in Figure 4, for a SNP with large effect size, for example, $\theta=0.7$, and MAF $=0.2$, the power of TSIR was close to 1 regardless of $\pi, \alpha_{1}$ and sample size $(\geqslant 500)$. Similarly, as seen in Figure 4 , for a SNP with a small effect size of $\theta=0.2$ and $\mathrm{MAF}=0.05$, the power of TSIR approach was close to 0 regardless of $\pi, \alpha_{1}$ and sample size $(\leqslant 2000)$. As seen with the binary phenotype, if $\alpha_{1}=10^{-3}, 10^{-4}$ and $10^{-5}$, with the increasing proportion $\pi$ of individuals included in step I, 'discovery cohort,' the power of TSIR first increased sharply then became plateaued around $\pi=0.6$ and 0.7 , which is particularly true for smaller values of $\alpha_{1}$.

Power comparisons among OS, TSIR, $T S_{1}$ and $T S_{2}$. It is clear from Figure 5 that, not surprisingly, the power of $\mathrm{TS}_{2}$ was larger owing to the fact that $\mathrm{TS}_{2}$ procedure used more individuals, and the power of $\mathrm{TS}_{1}$ was lower than OS even though $\mathrm{TS}_{1}$ uses the same number of individuals as OS but, under OS procedure, the analysis is conducted only once. The power of $\mathrm{TS}_{2}$ was larger than that of the TSIR approach especially when the sample size and effect size were moderate. However, this has to be balanced by the fact that the TS procedure used 30\% more individuals (for the validation cohort) than those used for TSIR approach. For the binary phenotype, the largest difference in power estimates between both approaches was seen to be 0.14 when there were 560 cases and 1400 controls corresponding to a SNP with MAF of 0.2 and effect size of 0.4 . For other situations, corresponding to large effect sizes or small/large sample sizes, the power estimates for the two approaches were comparable and reasonably close. The power of TSIR was almost identical to that of OS, which is expected, because for TSIR and OS, the sample was the same but TSIR used a re-sampling statistical technique to better control possible false positives (the simulated type I error rate of TSIR was smaller than $\alpha_{1} \times \alpha_{2}$ which is the theoretical type I error rate for OS) at the same time without sacrificing the power as $\mathrm{TS}_{1}$ did.

All simulation results for $\mathrm{TS}_{1}, \mathrm{TS}_{2}$ and TSIR were conducted using the twosided test in Stage II for $\mathrm{TS}_{1}$ and $\mathrm{TS}_{2}$ or in step 2 for TSIR, which will have slightly reduced power because it ignores the direction of association. We re-ran all simulations using exactly the same parameters as those for Figure 5 and re-calculated the power for $\mathrm{TS}_{1}, \mathrm{TS}_{2}$ and TSIR but used one-sided test in Stage II or step 2. For the binary phenotype, the maximum gain in power for TSIR with one-sided test was 0.01 . But for the $\mathrm{TS}_{1}$ and $\mathrm{TS}_{2}$, the maximum gain in power was 0.052 and 0.053 , respectively. The very similar conclusions held for continuous phenotype. One-sided test did improve the power of TSIR, but the power increase was relatively small which means the TSIR approach is relatively robust to one-sided or two-sided test owing to 100-round iterative resampling. For $\mathrm{TS}_{1}$ and $\mathrm{TS}_{2}$, though one-sided test improved their power at about $5 \%$, our simulations suggest that, in general, $\mathrm{TS}_{1}$ had smaller power than TSIR and $\mathrm{TS}_{2}$, but $\mathrm{TS}_{2}$ is not feasible owing to the lack of availability of an external validation cohort. Thus, the results further confirm the good performance and the practical usefulness of TSIR compared with OS or TS with or without the availability of additional validation cohort in ongoing and future GWAS or NGS.

Simulation studies for the obesity SNPs. Simulation studies were also conducted to estimate the empirical power for detecting association between SNPs identified for the obesity phenotype in Table 2 using the TSIR approach. ${ }^{10}$ The simulation parameters were taken to reflect the MAF, prevalence and effect size (in terms of odds ratios) observed in a cohort of cancer survivors exposed to cranial radiation therapy cohort (Table 2). Specifically, for each SNP, we first generated genotype data under Hardy-Weinberg equilibrium with MAF similar to that observed in the survivor cohort for a population with 2000000 individuals as above; and then, generated phenotype (case-control) data from the generated genotype dataset using the model above with odds ratio and prevalence of the disease same as those observed for the survivor cohort. Finally, a sample of 365 cases and 411 controls was randomly drawn from the population and analyzed using the TSIR approach. This process was repeated 10000000 and 10000 times for the estimation of empirical type I error and power, respectively. The empirical type I error rate was estimated as the proportion of times the SNP associated with obesity was validated wrongly and the empirical power was estimated as the proportion of times the SNP was validated correctly. For example, for SNP rs2769921 with MAF of 0.43, there was $69 \%$ power using the TSIR approach to detect whether the SNP was truly associated with obesity in cancer survivors with an odds ratio of 0.577 ; however, there was only $3.4 \times 10^{-6}$ chance to wrongly identify that this SNP was associated with obesity in cancer survivors (Table 2). Similarly, for SNP rs4971486 with MAF of 0.22 , the power to detect it was 0.69 if it was truly associated with obesity with an odds ratio of 1.9 and the type I error was $4.51 \times 10^{-6}$ if it were not associated with obesity.

\section{DISCUSSION}

It is well recognized that the top signals emerging from GWAS or next-generation sequencing must be validated in independent cohorts. ${ }^{19,20}$ However, independent external validation cohorts among those with rare diseases can be difficult to find within a limited timeframe. In such situations, TS resampling approaches have been used to identify and validate the SNPs associated with binary phenotypes of interest if the number of cases is not small. One such approach, namely the TSIR approach, has been proposed and we evaluated its operating characteristics through extensive simulation studies. These studies suggest that the TSIR approach, with the choice of 7:3 partitioning of the original cohort into 'discovery' and 'replication' cohorts, a cutoff of $r=20$ for identifying SNPs associated with the phenotype in 100 replications, and strictly controlling the type I error rate below $\alpha_{1} \times \alpha_{2}$ provide good type I error control and near optimal power. In our analyses, using the parameters above the power of the TSIR approach was found to be slightly lower than that observed for the $\mathrm{TS}_{2}$ approach, but this is owing to the fact that fewer individuals were included in the analyses of the TSIR approach than in the TS approach. Interestingly, with same sample sizes, the power of the TSIR was almost identical to that of OS, but the TSIR approach had a conservative type I error control than OS. It is often not possible to obtain an external cohort for validation for very rare diseases and unique cohorts. Thus, based on our analyses, we recommend the use of the TSIR approach for identifying the top candidate SNPs associated with a particular phenotype of interest. Identification of SNPs using the TSIR approach may help prioritize those candidate SNPs that should be evaluated in laboratory studies. However, it should be noted 
a $\mathrm{MAF}=0.05 ;$ prev $=0.3 ; \theta=0.2$

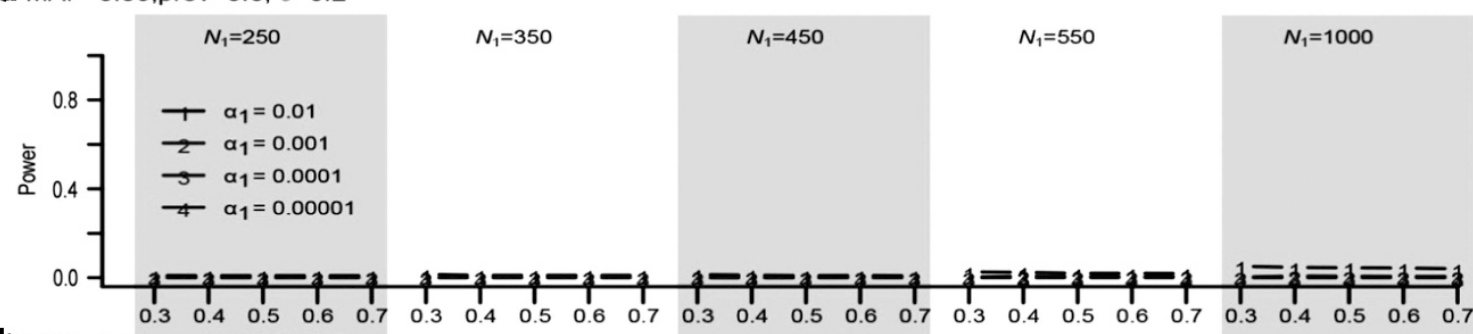

b $M A F=0.05 ;$ prev $=0.3 ; \theta=0.4$

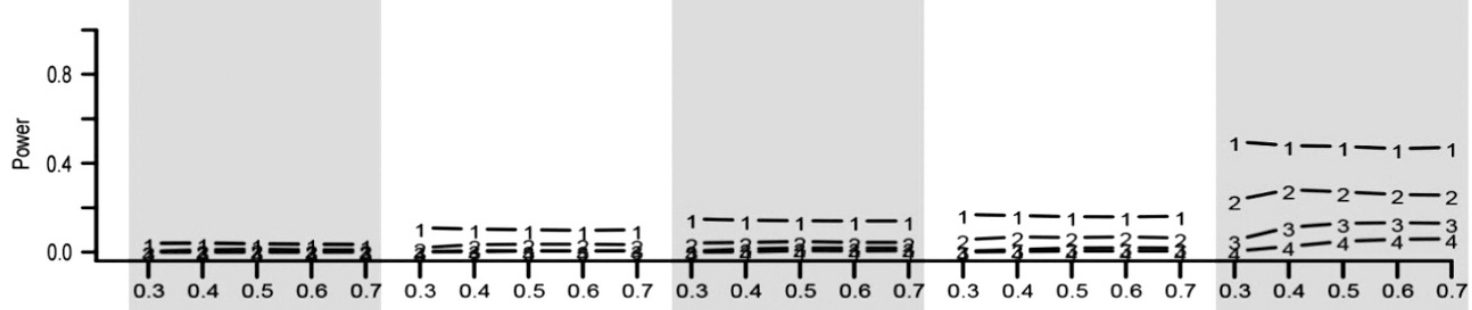

C $M A F=0.05 ;$ prev $=0.3 ; \theta=0.7$

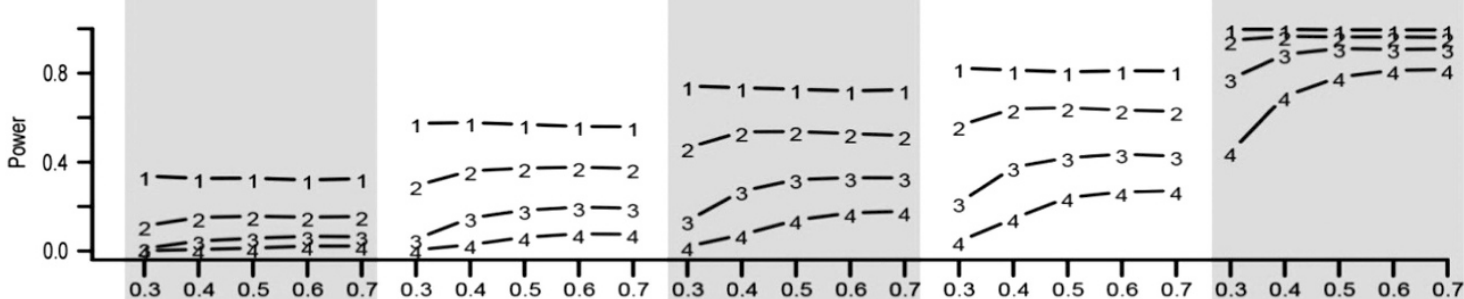

d $\mathrm{MAF}=0.05 ;$ prev $=0.01 ; \theta=0.2$

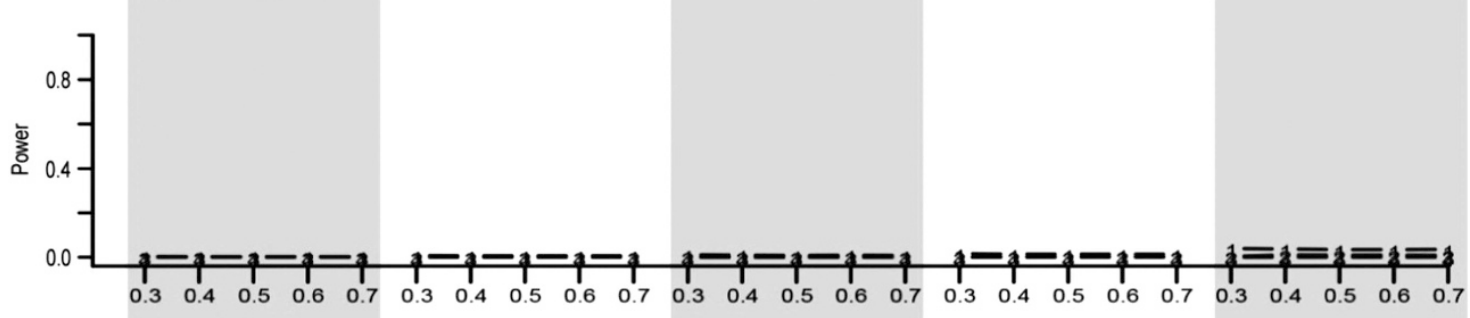

e $M A F=0.05 ;$ prev $=0.01 ; \theta=0.4$

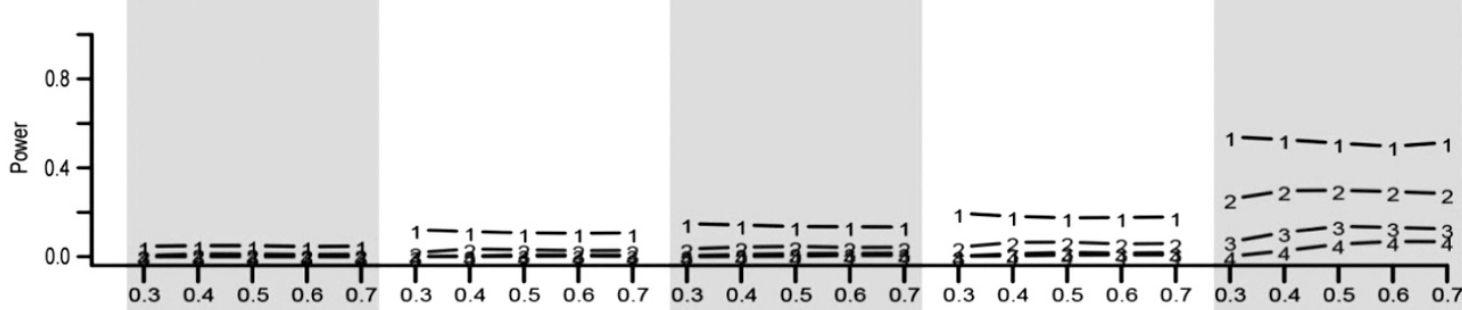

f $M A F=0.05 ;$ prev $=0.01 ; \theta=0.7$

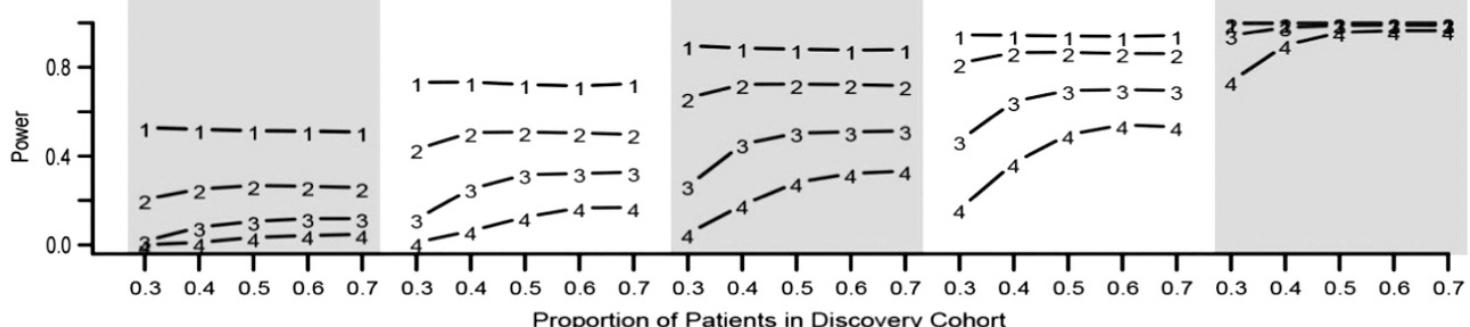

Figure 3 Empirical power of TSIR for detecting a SNP with a MAF of 0.05 for a binary phenotype. a-c are for small effect size $\theta=0.2$, moderate effect size 0.4 and large effect size 0.7 for a large prevalence of 0.3 , respectively. $\mathbf{d}-\mathbf{f}$ are for small effect size $\theta=0.2$, moderate effect size 0.4 and large effect size 0.7 for a small prevalence of 0.01 , respectively. The solid lines with the numbers of $1-4$ correspond to $\alpha_{1}=0.01,0.001,0.0001$ and 0.00001 , respectively. 
a $\mathrm{MAF}=0.2 ; \theta=0.2$

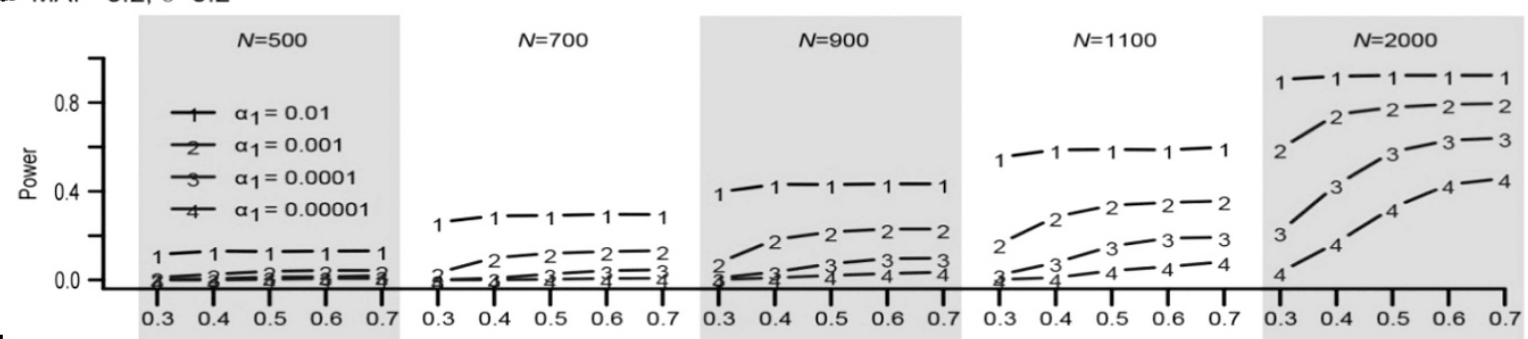

b $M A F=0.2 ; \theta=0.4$

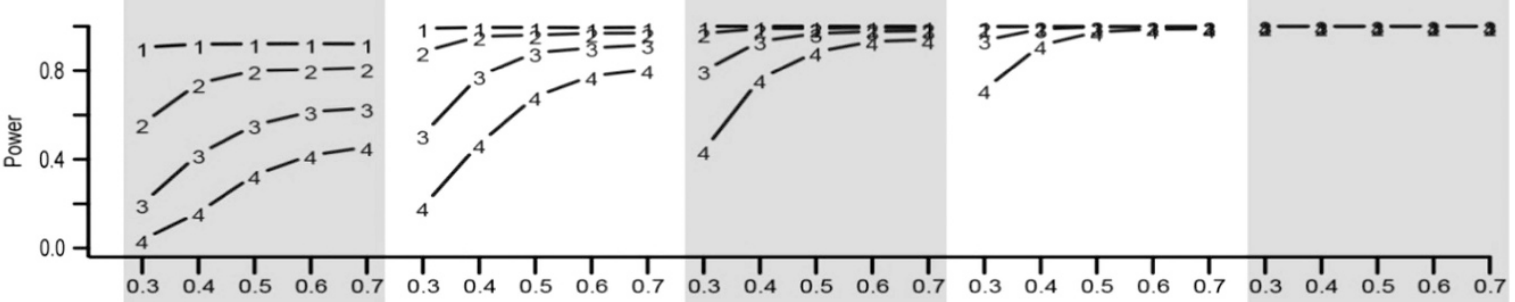

C $M A F=0.2 ; \theta=0.7$

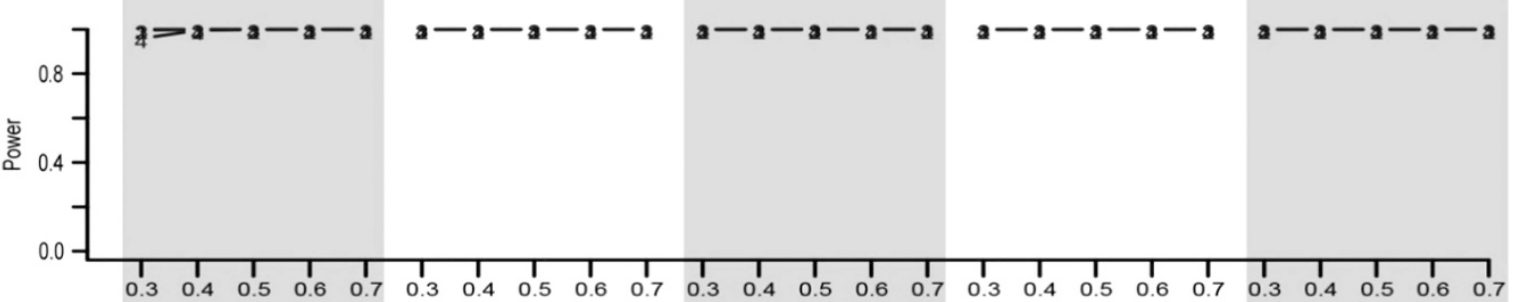

d $M A F=0.05 ; \theta=0.2$

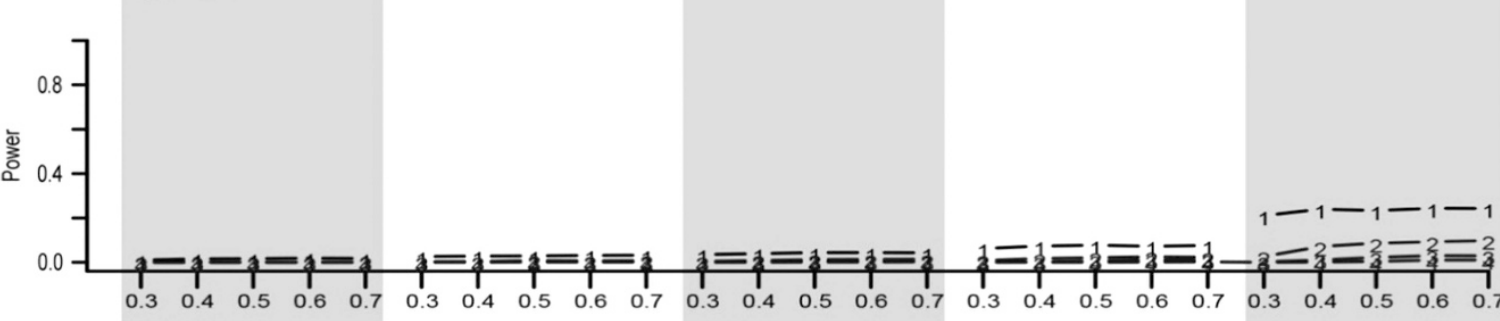

e $M A F=0.05 ; \theta=0.4$

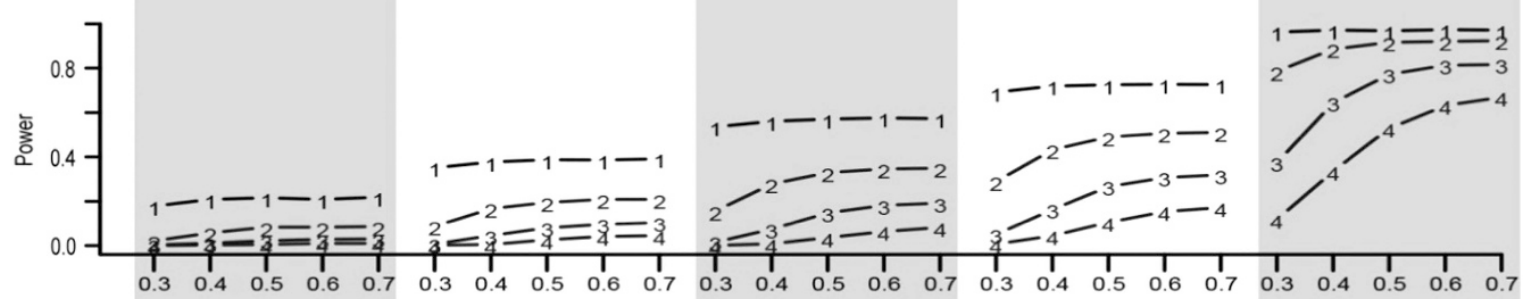

f $\mathrm{MAF}=0.05 ; \theta=0.7$

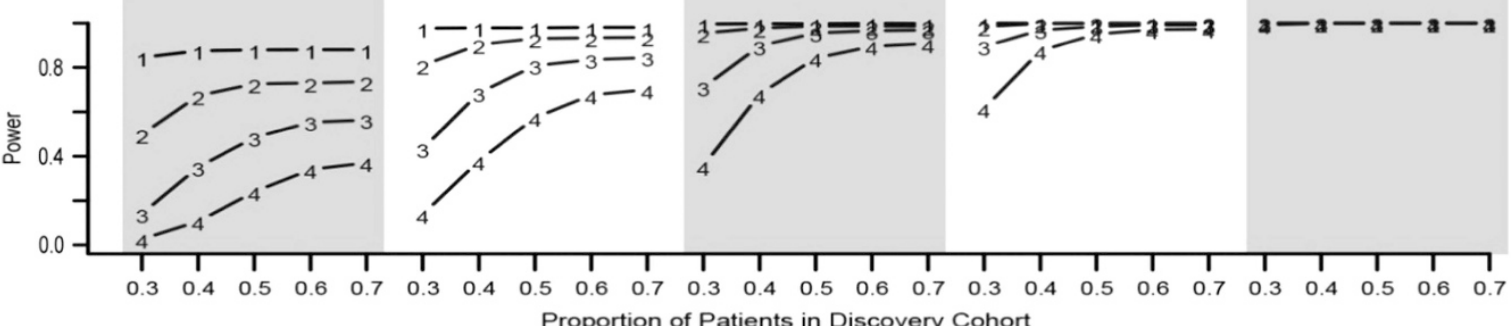

Figure 4 Empirical power of TSIR for detecting a SNP with a MAF of $0.05(\mathbf{a}-\mathbf{c})$ and $0.2(\mathbf{d}, \mathbf{e})$ for a continuous phenotype. $\mathbf{a}, \mathbf{b}$ and $\mathbf{c}$ are for small effect size $\theta=0.2$, moderate effect size 0.4 and large effect size 0.7 , respectively. The solid lines with the numbers of $1-4$ correspond to $\alpha_{1}=0.01,0.001$, 0.0001 and 0.00001 , respectively. 
a

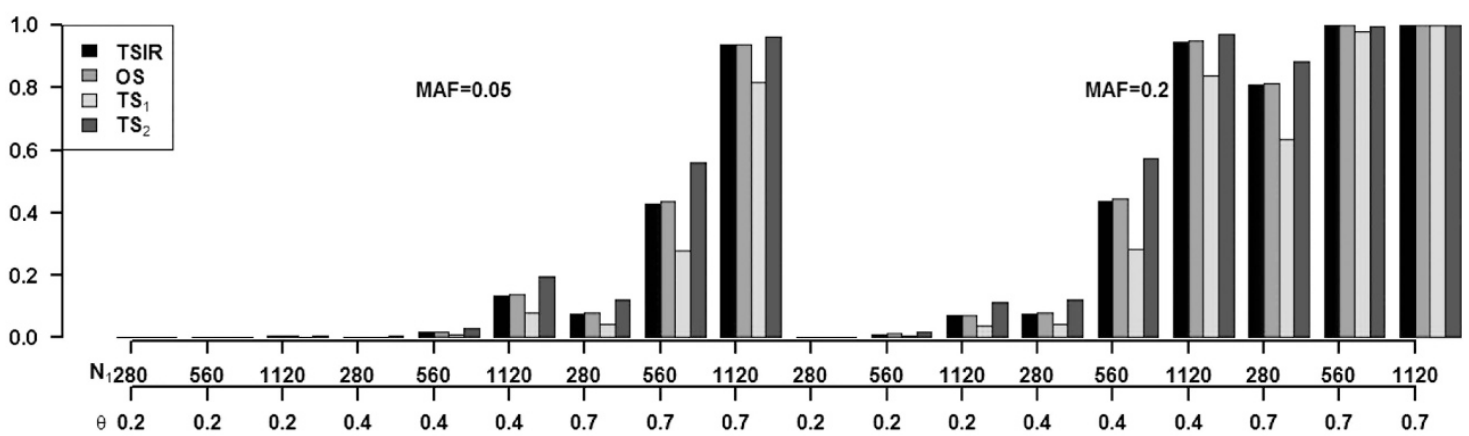

b

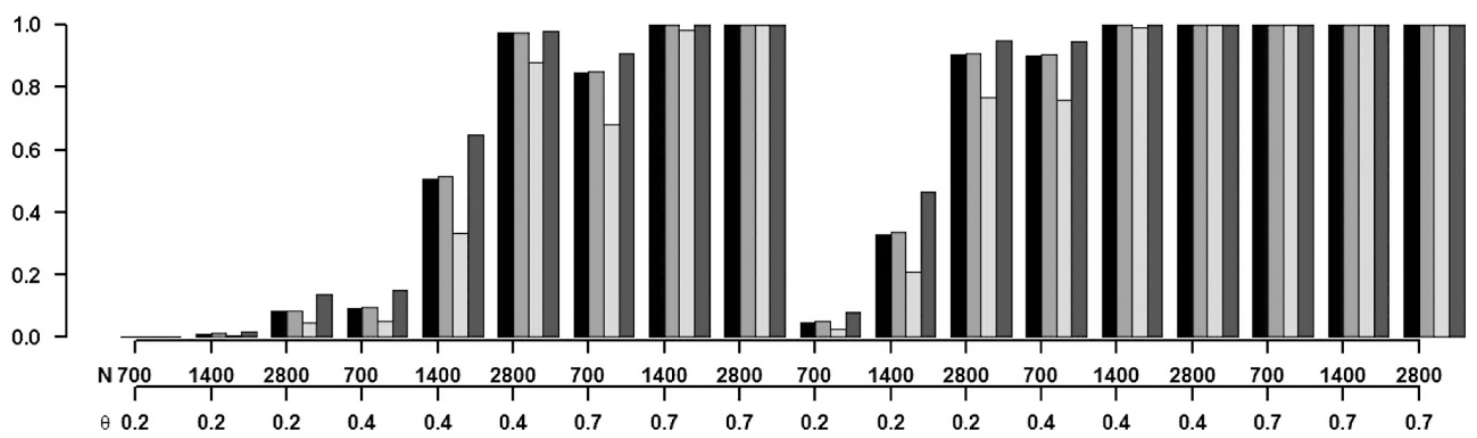

Figure 5 Power comparisons between TSIR, OS and TSs for detecting a SNP with MAFs of 0.05 and 0.2 associated with binary (a) and continuous (b) phenotypes. The first $x$ axis for $\mathbf{a}$ and $\mathbf{b}$ is for the number of cases (the number of controls is 2.5 times of the number of cases) and the number of individuals, respectively. The second $x$ axis is for $\theta$. The four bars are for TSIR, OS, $\mathrm{TS}_{1}$ and $\mathrm{TS}_{2}$, respectively. A full color version of this figure is available at the Journal of Human Genetics journal online.

Table 2 Empirical power and type I error rate $\left(\times 10^{6}\right)$ simulation results for 21 SNPs identified associated with BMI

\begin{tabular}{|c|c|c|c|c|c|c|c|}
\hline rs4971486 & 2 & 4895318 & G & 1.936 & 0.2223 & 0.686 & 4.51 \\
\hline rs6745523 & 2 & 4908703 & $A$ & 1.939 & 0.1768 & 0.518 & 3.30 \\
\hline rs12648678 & 4 & 175598280 & G & 0.5091 & 0.1699 & 0.516 & 3.70 \\
\hline rs2171139 & 4 & 175624314 & $C$ & 0.5196 & 0.1735 & 0.503 & 3.80 \\
\hline rs2443547 & 5 & 18173672 & C & 0.5911 & 0.4329 & 0.621 & 3.90 \\
\hline rs2923756 & 5 & 18192581 & G & 1.836 & 0.1972 & 0.443 & 3.40 \\
\hline rs12514191 & 5 & 18198169 & G & 1.804 & 0.194 & 0.404 & 2.90 \\
\hline rs315825 & 5 & 18198934 & $A$ & 0.595 & 0.4334 & 0.612 & 2.91 \\
\hline rs2938412 & 5 & 18205107 & A & 1.603 & 0.4472 & 0.434 & 4.20 \\
\hline rs453891 & 5 & 18212493 & $\mathrm{~T}$ & 0.5946 & 0.4361 & 0.606 & 3.60 \\
\hline rs1316610 & 5 & 18230131 & $C$ & 1.835 & 0.1893 & 0.453 & 2.60 \\
\hline rs2972911 & 5 & 18258703 & $A$ & 0.5932 & 0.4432 & 0.605 & 2.70 \\
\hline rs2019973 & 5 & 134573992 & $T$ & 0.6216 & 0.4691 & 0.441 & 3.41 \\
\hline rs2769921 & 13 & 107794883 & $C$ & 0.577 & 0.43 & 0.690 & 3.40 \\
\hline rs12709954 & 19 & 56716579 & $\mathrm{~T}$ & 0.5784 & 0.2874 & 0.515 & 3.22 \\
\hline
\end{tabular}

Abbreviations: BMI, body mass index; Chr, chromosome; MA, minor allele; MAF, minor allele frequency; OR, odds ratio; SNP, single nucleotide polymorphism.

aSNP identifier according to the dbSNP database.

bPhysical location of SNP based on human gene assembly 19. 
that the TSIR approach is only applicable when the size of the population of interest is sufficiently large for sample splitting.

In GWAS, the first step prior to statistical genetic association testing is quality control analysis which includes Hardy-Weinburg disequilibrium test to remove markers departing from Hardy-Weinberg equilibrium. ${ }^{21}$ Thus, in our TSIR simulations, the genotype data are generated by assuming Hardy-Weinberg equilibrium. However, if we are concerned about Hardy-Weinburg disequilibrium in a GWAS, then some statistical association testing method ${ }^{22}$ that can adjust for Hardy-Weinburg disequilibrium is available and can be used to replace the logistic regression in TSIR, but we would expect the conclusions drawn above would still hold. Furthermore, in our simulations, we used logistic regression. In the literature, there are many statistical methods available for genetic association testing, which can also be used in the TSIR approach. ${ }^{19}$ We would expect that the conclusions drawn above would still hold. The common SNPs with MAFs of 0.2 and 0.05 in GWAS were investigated in this study. Currently, rare variant association identification in the next-generation sequencing studies is highly in demand owing to missing inheritability of complex trait post-GWAS. ${ }^{23}$ If the sample size of the study is large enough so that the splitting of the cohort is reasonable, then the TSIR approach allows for sufficient statistical power to detect the rare variants in both steps. ${ }^{24}$ With smaller sample sizes where splitting is unreasonable, a permutation test may be applied. However, for rare variant association, we often conduct gene (set)-based analysis, ${ }^{18,25}$ not single SNP-based analysis. This way we can use the TSIR procedure as an internal validation method if there is no external validation cohort available.

If the individuals in the study cohort are from different populations, we can just simply adjust for population stratification by including genetic ancestry score as covariates in the logistic regression model. ${ }^{26}$ Here, our interest was on detecting genetic effect on the binary outcome. In post-GWAS, besides rare variant associations above, gene-environment interaction also has an important role in finding missing inheritability for complex trait. ${ }^{27}$ They are worthy of investigation by simulations, but we would expect that similar conclusions would hold.

\section{CONFLICT OF INTEREST}

The authors declare no conflict of interest.

\section{ACKNOWLEDGEMENTS}

We would like to thank two reviewers for their helpful comments which have significantly improved the paper. This research was supported by St. Jude Children's Research Hospital Cancer Center Support (CORE) grant CA21765 from the National Cancer Institute and by the American Lebanese and Syrian Associated Charities (ALSAC). The research work of Jun J Yang was in part supported by the grant U01CA176063.

1 Klein, R. J., Zeiss, C., Chew, E. Y., Tsai, J. Y., Sackler, R. S., Haynes, C. et al. Complement factor $\mathrm{H}$ polymorphism in age-related macular degeneration. Science $\mathbf{3 0 8}$ 385-389 (2005)

2 Sladek, R., Rocheleau, G., Rung, J., Dina, C., Shen, L., Serre, D. et al. A genome-wide association study identifies novel risk loci for type 2 diabetes. Nature 445 , 881-885 (2007)
3 The Wellcome Trust Case Control Consortium (WTCCC). Genome-wide association study of 14,000 cases of seven common diseases and 3,000 shared controls. Nature 447 , 661-678 (2007).

4 Skol, A. D., Scott, L. J., Abecasis, G. R. \& Boehnke, M. Joint analysis is more efficient than replication-based analysis for two-stage genome-wide association studies. Nat. Genet. 38, 209-213 (2006).

5 Pahl, R., Schäfer, H. \& Müller, H.-H. Optimal multistage designs-a general framework for efficient genome-wide association studies. Biostatistics 10, 297-309 (2008).

6 Rothman, N., Garcia-Closas, M., Chatterjee, N., Malats, N., Wu, X., Figueroa, J. D. et al. A multi-stage genome-wide association study of bladder cancer identifies multiple susceptibility loci. Nat. Genet. 42, 978-984 (2010)

7 Gurney, J. G., Severson, R. K., Davis, S. \& Robison, L. L. Incidence of cancer in children in the United States. Sex-, race-, and 1-year age-specific rates by histologic type. Cancer 75, 2186-2195 (1995)

8 Wheeler, H. E., Maitland, M. L., Dolan, M. E., Cox, N. J. \& Ratain, M. J. Cance pharmacogenomics: strategies and challenges. Nat. Rev. Genet. 14, 23-34 (2013).

9 Hudson, M. M., Ness, K. K., Nolan, V. G., Armstrong, G. T., Green, D. M., Morris, E. B. et al. Prospective medical assessment of adults surviving childhood cancer: study design, cohort characteristics, and feasibility of the St. Jude Lifetime Cohort Study. Pediatr. Blood Cancer 56, 825-836 (2011)

10 Wilson, C. L., Liu, L., Yang, J. J., Kang, G., Ojha, R. P., Neale, G. et al. Genetic and clinical factors associated with obesity among adult survivors of childhood cancer a report from the St. Jude Lifetime cohort. Cancer (e-pub ahead of print 11 May 2015)

11 Yang, J. J., Cheng, C., Devidas, M., Cao, X., Campana, D., Yang, W. et al. Genome-wide association study identifies germline polymorphisms associated with relapse of childhood acute lymphoblastic leukemia. Blood 120, 4197-4204 (2012)

12 Elliott, K. S., Chapman, K., Day-Williams, A., Panoutsopoulou, K., Southam, L., Lindgren, C. M. et al. GIANT consortium Evaluation of the genetic overlap between osteoarthritis with body mass index and height using genome-wide association scan data. Ann. Rheum. Dis. 72, 935-941 (2013)

13 Hayes, M. G., Pluzhnikov, A., Miyake, K., Sun, Y., Ng, M. C., Roe, C. A. et al Identification of type 2 diabetes genes in Mexican Americans through genome-wide association studies. Diabetes 56, 3033-3044 (2007)

$14 \mathrm{Cheng}, \mathrm{C}$. Internal validation inferences of significant genomic features in genome-wide screening. Comput. Stat. Data Anal. 53, 788-800 (2009).

15 Simón-Sánchez, J., Schulte, C., Bras, J. M., Sharma, M. Gibbs, J. R., Berg, D. et al. Genome-wide association study reveals genetic risk underlying Parkinson's disease. Nat. Genet. 41, 1308-1312 (2009)

16 Yue, W. H., Wang, H. F., Sun, L. D., Tang, F. L., Liu, Z. H., Zhang, H. X. et al. Genomewide association study identifies a susceptibility locus for schizophrenia in Han Chinese at 11p11.2. Nat. Genet. 43, 1228-1231 (2011)

17 Kang, G., Bi, W., Zhao, Y., Zhang, J. F., Yang, J. J., Xu, H. et al. A new system identification approach to identify genetic variants in sequencing studies for a binary phenotype. Hum. Hered. 78, 104-116 (2014)

18 Wu, M. C., Lee, S., Cai, T., Li, Y., Boehnke, M. \& Lin, X. Rare-variant association testing for sequencing data with the sequence kernel association test. Am. J. Hum. Genet. 89 82-93 (2011).

19 Igl, B. W., Konig, I. R. \& Ziegler, A. What do we mean by 'replication' and 'validation' in genome-wide association studies? Hum. Hered. 67, 66-68 (2009).

20 loannidis, J. P. A., Gilles, T. \& Daly, M. J. Validating, augmenting and refining genomewide association signals. Nat. Rev. Genet. 10, 318-329 (2009).

21 Purcell, S., Neale, B., Todd-Brown, K., Thomas, L., Ferreira, M. A., Bender, D. et al. PLINK: a toolset for whole-genome association and population-based linkage analysis. Am. J. Hum. Genet. 81, 559-575 (2007)

22 Song, K. \& Elston, R. C. A powerful method of combining measures of association and Hardy-Weinberg disequilibrium for fine-mapping in case-control studies. Stat. Med. 25, 105-126 (2006).

23 Freidlin, B., Zheng, G., Li, Z. \& Gastwirth, J. L. Trend tests for case-control studies of genetic markers: power, sample size and robustness. Hum. Hered. 53 146-152 (2002) Erratum in Hum Hered 2009; 68: 220.

24 Kang, G., Lin, D., Hakonarson, H. \& Chen, J. Two-stage extreme phenotype sequencing design for discovering and testing common and rare genetic variants: efficiency and power. Hum. Hered. 73, 139-147 (2012).

25 Lee, S., Emond, M. J., Bamshad, M. J., Barnes, K. C., Rieder, M. J., Nickerson, D. A. et al NHLBI GO Exome Sequencing Project-ESP Lung Project Team Optimal unified approach for rare-variant association testing with application to small-sample case-control wholeexome sequencing studies. Am. J. Hum. Genet. 91, 224-237 (2012)

26 Kang, G., Gao, G., Shete, S., Redden, D. T., Chang, B.-L., Rebbeck, T. R. et al. Capitalizing on admixture in genome-wide association studies: A two-stage testing procedure and application to height in African-Americans. Front. Genet. 2 11 (2011)

27 Chen, J., Kang, G., VanderWeele, T., Zhang, C. \& Mukherjee, B. Efficient designs of gene-environment interaction studies: implications of Hardy-Weinberg equilibrium and gene-environment independence. Stat. Med. 31, 2516-2530 (2012).

Supplementary Information accompanies the paper on Journal of Human Genetics website (http://www.nature.com/jhg) 